\title{
cIAP1 and TAK1 protect cells from TNF-induced necrosis by preventing RIP1/RIP3-dependent reactive oxygen species production
}

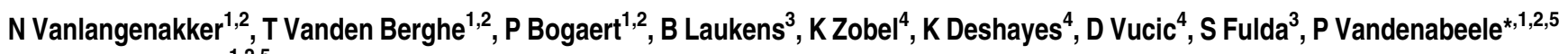 \\ and MJM Bertrand ${ }^{1,2,5}$
}

Three members of the IAP family (X-linked inhibitor of apoptosis (XIAP), cellular inhibitor of apoptosis proteins-1/-2 (cIAP1 and cIAP2)) are potent suppressors of apoptosis. Recent studies have shown that cIAP1 and cIAP2, unlike XIAP, are not direct caspase inhibitors, but block apoptosis by functioning as E3 ligases for effector caspases and receptor-interacting protein 1 (RIP1). clAP-mediated polyubiquitination of RIP1 allows it to bind to the pro-survival kinase transforming growth factor$\beta$-activated kinase 1 (TAK1) which prevents it from activating caspase-8-dependent death, a process reverted by the deubiquitinase CYLD. RIP1 is also a regulator of necrosis, a caspase-independent type of cell death. Here, we show that cells depleted of the IAPs by treatment with the IAP antagonist BV6 are greatly sensitized to tumor necrosis factor (TNF)-induced necrosis, but not to necrotic death induced by anti-Fas, poly(I:C) oxidative stress. Specific targeting of the IAPs by RNAi revealed that repression of cIAP1 is responsible for the sensitization. Similarly, lowering TAK1 levels or inhibiting its kinase activity sensitized cells to TNF-induced necrosis, whereas repressing CYLD had the opposite effect. We show that this sensitization to death is accompanied by enhanced RIP1 kinase activity, increased recruitment of RIP1 to Fas-associated via death domain and RIP3 (which allows necrosome formation), and elevated RIP1 kinase-dependent accumulation of reactive oxygen species (ROS). In conclusion, our data indicate that cIAP1 and TAK1 protect cells from TNF-induced necrosis by preventing RIP1/RIP3-dependent ROS production.

Cell Death and Differentiation (2011) 18, 656-665; doi:10.1038/cdd.2010.138; published online 5 November 2010

Until recently, necrosis was defined, in contrast to apoptosis, as an accidental and uncontrolled type of cell death. This definition is now obsolete, because accumulating experimental evidence has shown that necrosis is a well-regulated process activated by rather specific physiological and pathological stimuli. ${ }^{1}$ Necroptosis, or programmed necrosis, is a caspase-independent mode of cell death that prevails when caspases are either not activated or inhibited. ${ }^{2}$ The term 'necroptosis' has been confined to regulated necrotic cell death mediated by receptor-interacting protein 1 (RIP1) kinase activity, and so by definition it is inhibited by necrostatin-1 (Nec-1). ${ }^{3,4}$ It might be inappropriate, however, to restrict the definition of a cellular process to the activation of a particular initiator, as different pathways could initiate the process of necrosis. ${ }^{5}$ Necrosis occurs in medical conditions such as ischemia-reperfusion damage during organ transplantation, cardiac infarction, stroke, neurodegenerative diseases, and viral infection. ${ }^{6}$ Little is known about the proteins involved in and regulating signaling during programmed necrosis. Recent reports have identified two members of the serine-threonine RIP kinase family, RIP1 and RIP3, as crucial components of the tumor necrosis factor (TNF)-mediated necrotic process. ${ }^{7-10}$ Interestingly, RIP1 has also been implicated in the apoptotic process triggered by several death receptors. ${ }^{11}$ This suggests that apoptosis and necrosis, initially defined as mutually exclusive cell death processes, might paradoxically share several regulatory components.

Among the arsenal of proteins that regulate the apoptotic process are the members of the inhibitor of apoptosis protein (IAP) family, which are potent suppressors of apoptotic cell death. It has long been assumed that their protective effect was exclusively due to their ability to bind and inhibit caspases, but recent findings have challenged this view. Structure-function studies have shown that, among the eight IAPs contained in the human genome, X-linked inhibitor of

\footnotetext{
${ }^{1}$ Department for Molecular Biomedical Research, VIB, Technologiepark 927, Zwijnaarde-Ghent 9052, Belgium; ${ }^{2}$ Department of Biomedical Molecular Biology, Ghent University, Technologiepark 927, Zwijnaarde-Ghent 9052, Belgium; ${ }^{3}$ Institute for Experimental Cancer Research in Pediatrics, Goethe-University Frankfurt, Komturstr. 3a, Frankfurt 60528, Germany and ${ }^{4}$ Genentech Inc., 1 DNA Way, South San Francisco, CA 94080, USA

*Corresponding author: P Vandenabeele, Department for Molecular Biomedical Research, VIB-Ghent University, Technologiepark 927, 9000 Ghent, Belgium.

Tel: + 320933 13760; Fax: + 320933 13609; E-mail: Peter.Vandenabeele@dmbr.vib-UGent.be

${ }^{5}$ These authors contributed equally to this work.

Keywords: TNFR1; necrosis; smac mimetic; TAK1; cIAP1; necrosome

Abbreviations: 5Z-7, 5Z-7-oxozeaenol; BHA, butylated hydroxyanisole; cIAP1/2, cellular inhibitor of apoptosis proteins-1/-2; CYLD, cylindromatosis; DHR123, dihydrorhodamine 123; ERK, extracellular signal-regulated kinase; FADD, Fas-associated via death domain; $\mathrm{H}_{2} \mathrm{O}_{2}$, hydrogen peroxide; IFN $\beta$, interferon- $\beta$; MEK1/2, mitogen-activated protein kinase kinase 1/-2; NDUFB8, NADH dehydrogenase (ubiquinone) 1 beta subcomplex 8; Nec-1, necrostatin-1; Nox1, NADPH oxidase 1; RFK, riboflavin kinase; RIP, receptor-interacting protein; ROS, reactive oxygen species; TAK1, transforming growth factor- $\beta$-activated kinase 1; TLR3, toll-like receptor 3; TNF, tumor necrosis factor; TNFR1, tumor necrosis factor receptor 1; XIAP, X-linked inhibitor of apoptosis; zVAD-fmk, benzyloxycarbonyl-Val-Ala-Asp(OMe)fluoromethylketone

Received 30.4.10; revised 10.9.10; accepted 24.9.10; Edited by M Deshmukh; published online 05.11.10
} 
apoptosis (XIAP) is the only IAP that acts as a direct caspase inhibitor. ${ }^{12}$ In contrast, cellular inhibitor of apoptosis proteins-1 and -2 (cIAP1 and clAP2) were shown to protect cells from apoptosis by acting as E3 ubiquitin ligases, an enzymatic activity conferred by their C-terminal RING finger domains. Protein ubiquitination is emerging as a key regulatory mechanism and its wide range of action depends on the ability of ubiquitin to form polymers: Lys48-linked ubiquitin chains typically target proteins for proteasomal degradation, while Lys63-linked chains serve as a docking site for signaling complexes. The role of clAPs as E3 ligases in apoptosis regulation is complex and implicates both Lys48- and Lys63polyubiquitination. On the one hand, clAP1 mediates proteasomal degradation of caspase-3 and $-7^{13}$ and of $\mathrm{NF}-\kappa \mathrm{B}$-inducing kinase $^{14,15}$ On the other hand, clAPmediated Lys63-polyubiquitination of RIP1 allows it to bind to the pro-survival kinase transforming growth factor- $\beta$ activated kinase 1 (TAK1), which prevents it from activating a pro-apoptotic complex made of RIP1 and caspase-8. ${ }^{16}$ Accordingly, cylindromatosis (CYLD), a de-ubiquitinase that specifically hydrolyzes Lys63-linked polyubiquitin chains, counteracts the pro-survival effects of cIAPs on RIP1. ${ }^{17,18}$

The observation that clAPs are potent suppressors of cell death and that they mediate this function, at least in part, by a mechanism that does not involve direct caspase inhibition but entails modulation of RIP1 ubiquitination status, indicates that clAPs and TAK1 could play a role in necrosis signaling. In this study, we tested this idea and identified CIAP1 and TAK1 as new regulators of TNF-induced programmed necrosis.

\section{Results}

BV6 treatment sensitizes cells to necrosis induced by TNF, but not to necrosis induced by anti-Fas, poly(I:C), or hydrogen peroxide. A widely used cellular system for studying necrotic cell death is the mouse fibrosarcoma L929 cell line. In these cells, necrosis can be elicited by stimulation with TNF alone, ${ }^{2}$ a combination of Fas receptor agonistic antibodies and the pan-caspase inhibitor benzyloxycarbonylVal-Ala-Asp(OMe)-fluoromethylketone (zVAD-fmk), ${ }^{19}$ doublestranded RNA (poly $(\mathrm{l}: \mathrm{C})$ ) in the presence of interferon- $\beta$ $(\mathrm{IFN} \beta),{ }^{20}$ or by treating the cells with hydrogen peroxide $\left(\mathrm{H}_{2} \mathrm{O}_{2}\right){ }^{5}$ As a first approach to investigate the potential role of the clAPs in necrotic cell death, we used BV6, an IAP antagonist that induces autoubiquitination and subsequent proteasomal degradation of clAP1 and clAP2 (Figure 1a). ${ }^{15}$ Surprisingly, we found that BV6 treatment had no effect on necrotic cell death induced by $\operatorname{poly}(\mathrm{I}: \mathrm{C})+\operatorname{IFN} \beta$, antiFas + zVAD-fmk, or $\mathrm{H}_{2} \mathrm{O}_{2}$, but greatly sensitized cells to TNF-induced necrosis (Figure 1b). Importantly, BV6 exposure alone did not lead to cell death (Figure $1 \mathrm{~b}$ ), and sensitization to TNF was observed even at very low doses of TNF (Figure 1c). Interestingly, although treatment with the RIP1 kinase inhibitor Nec-1 could block necrosis induced by TNF, poly(I:C) + IFN $\beta$, and anti-Fas +zVAD-fmk (Supplementary Figure 1A), IAP depletion by BV6 could only sensitize cells to TNF-induced death. As reported previously, $\mathrm{H}_{2} \mathrm{O}_{2}$-induced necrosis could not be blocked by Nec-1. ${ }^{5}$ To test whether the enhanced cell death caused by TNF + BV6 was purely necrotic and not due to cumulative induction of apoptosis, we analyzed the processing of caspase-3, a clear marker of apoptosis. Caspase- 3 cleavage was not detected, even in conditions in which up to $83 \%$ of the cells died. In contrast, caspase-3 processing was observed in the anti-Fas-treated controls (Figure 1d). To determine whether BV6 sensitization to TNF-induced necrosis is restricted to L929 cells or also applies to other necrotic model systems, we tested the effect of BV6 on Jurkat T cells deficient in Fas-associated via death domain (FADD). The absence of FADD in these cells renders them resistant to TNF-induced apoptosis and directs the system to necrosis. As observed in L929 cells, BV6 treatment of FADD-deficient Jurkat cells led to a rapid loss of clAP1 and clAP2 and greatly sensitized these cells to TNF-induced necrosis (Figure 1e). Taken together, these results show that BV6 specifically sensitizes cells to TNF-induced necrosis, and that this effect is not restricted to L929 cells.

\section{Loss of clAP1 sensitizes L929 cells to TNF-induced} necrosis. To exclude the possibility that BV6 enhances TNF-induced necrosis through an off-target effect, we next investigated the effect of specific repression of cIAP1, clAP2, and XIAP in L929 cells by using an RNAi-based approach. Indeed, although BV6 treatment does not alter XIAP levels, recent studies have shown that the binding of BV6 to XIAP could abrogate its function. ${ }^{21}$ We tested the efficiency of clAP1 and clAP2 knockdowns by immunoblot using RIAP1, an antibody detecting both CIAP1 and clAP2. ${ }^{22}$ ClAP1 RNAi completely repressed the signal detected by RIAP1, but clAP2 RNAi did not alter it (Figure 2a), which shows that clAP2 protein levels are below the immunoblot detection limit in $L 929$ cells. We therefore confirmed clAP2 repression by RT-PCR (Figure 2b). Remarkably, we found that repression of clAP1 greatly increased TNF-induced necrotic cell death, whereas repression of clAP2 or XIAP had no effect (Figure 2c). These data clearly illustrate that clAP1 plays a protective role in TNF-induced necrosis in L929 cells and confirm that the BV6 effect is due to the loss of clAP1 function.

cIAP1 repression induces RIP1 kinase activity. Two members of the serine-threonine RIP kinase family, RIP1 and RIP3, are crucial components of the TNF-induced necrotic process, which according to recent studies requires active RIP1 kinase activity ${ }^{3,9}$ to allow formation of a RIP1-RIP3-FADD necrosome complex. ${ }^{7,8,10}$ To position clAP1 in the TNF necrotic pathway, we treated L929 cells with BV6 and Nec-1, alone or together, and then challenged them with a lethal dose of TNF for $4 \mathrm{~h}$. Nec-1 completely protected cells from TNF-induced necrosis, both in the absence and presence of BV6 (Figure 3a). This inhibitory effect was observed even after $24 \mathrm{~h}$ of TNF stimulation (Supplementary Figure 1B). In parallel experiments, we found that depleting RIP3 by an RNAi-based approach also abrogated BV6 sensitization to TNF-induced death, which places clAP1 upstream or at the level of RIP1 and RIP3 in the TNF necrotic pathway (Figure 3b). Next, by looking at extracellular signal-regulated kinase (ERK), ${ }^{23}$ we investigated whether clAP1 could influence RIP1 kinase activity. We observed that BV6 treatment greatly enhanced 


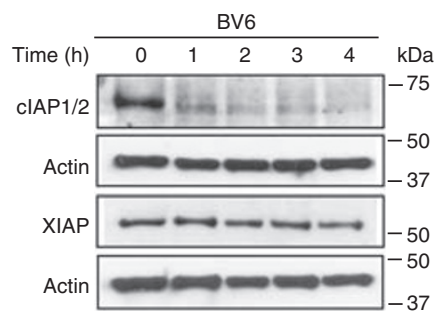

C

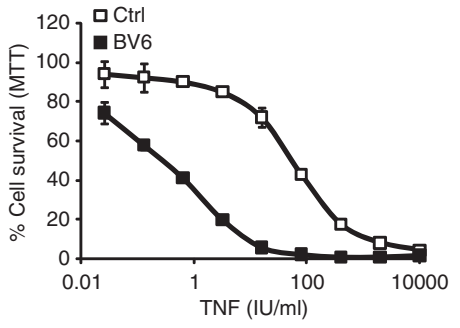

b
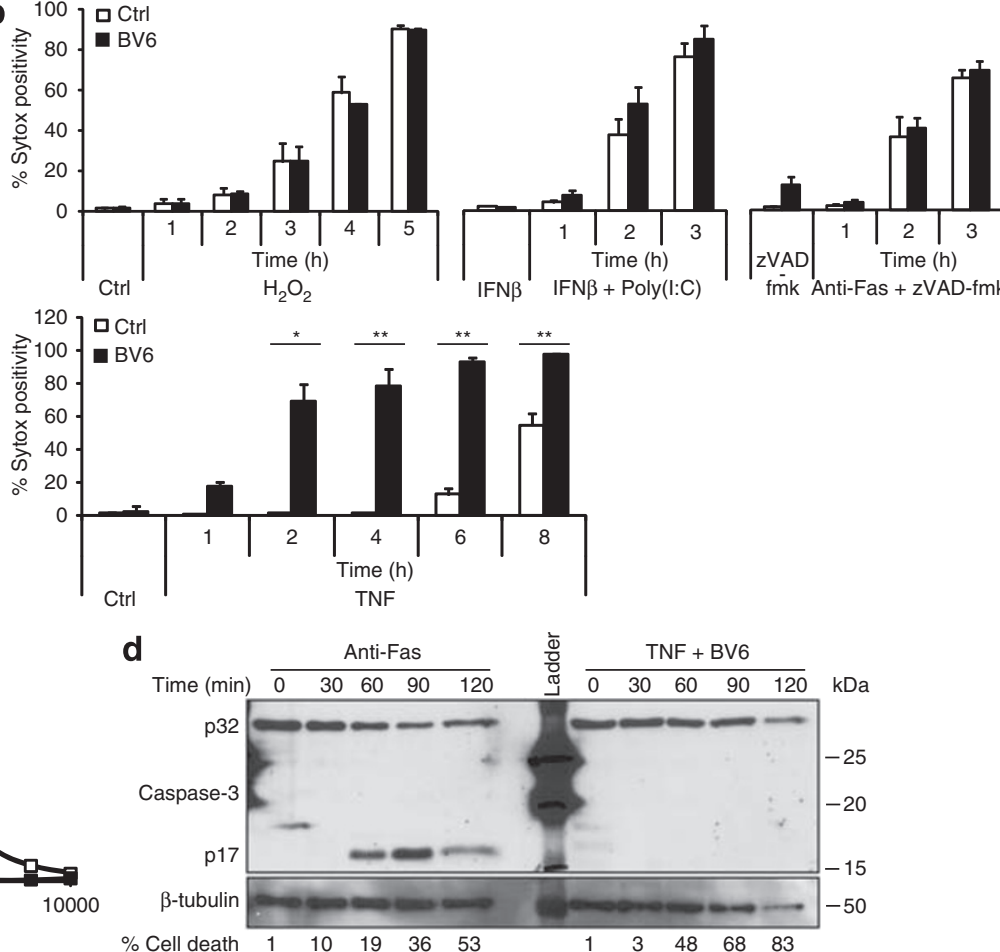

e
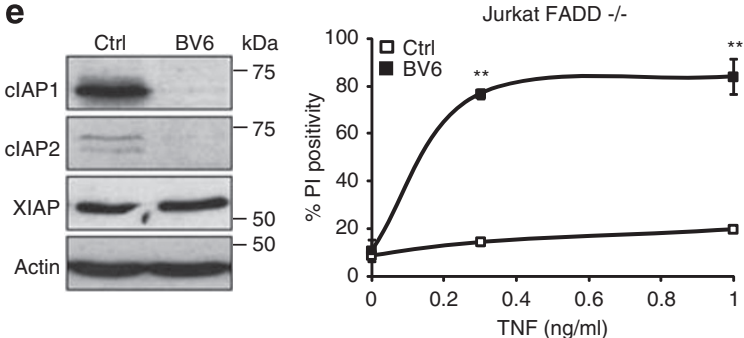

Figure 1 BV6 treatment sensitizes cells to necrosis induced by TNF, but not to necrosis induced by anti-Fas, poly(l:C), or $\mathrm{H}_{2} \mathrm{O}_{2}$. (a) $\mathrm{L}_{2} 29$ cells were treated with $1 \mu \mathrm{M}$ BV6 for the indicated durations. Cells were lysed and cIAP1/2 and XIAP were immunoblotted. (b) L929 cells were pre-treated with IFN $\beta$, zVAD-fmk, or medium in the presence of BV6, and then stimulated with the mentioned triggers for the indicated times. Cell death (\% Sytox positivity) was analyzed by flow cytometry. Data are representative of three independent experiments. Error bars represent standard deviation. (c) L929 cells were pre-treated with BV6 and stimulated with a serial dilution of TNF. After $20 \mathrm{~h}$, cell viability was determined by an MTT assay. Error bars represent standard deviations of triplicates. (d) L929 cells were pre-treated with BV6 and stimulated with TNF. Cell lysates were made, and processed caspase-3 was checked by western blot. Stimulation with anti-Fas was included as a positive control for apoptosis. Cell death was determined by flow cytometry. (e) FADD - / - Jurkat cells were pre-treated with BV6 and stimulated with TNF for $24 \mathrm{~h}$. Cell death (PI positivity) was analyzed by flow cytometry. Levels of clAP1, CIAP2 and XIAP were checked on western blot. ${ }^{*} P<0.05 ;{ }^{*} P<0.01$ (Mann-Whitney U-test)

TNF-induced ERK phosphorylation, and by using BV6 and Nec-1 together, we confirmed that this effect was RIP1 kinase dependent (Figure 3c). To rule out the possibility of off-target effects of these chemical agents, we confirmed the influence of clAP1 on RIP1 kinase-mediated ERK phosphorylation by specifically repressing ClAP1 and RIP1 (Figure 3d). To test if RIP1-dependent ERK phosphorylation plays a direct role in BV6 sensitization to TNF-induced necrosis, we compared cell death in the absence or presence of U0126, a chemical inhibitor of the ERK-activating kinase (mitogen-activated protein kinase kinase 1/2), but we did not observe significant differences (Supplementary Figure 2). The effect of clAP1 repression on RIP1 kinase activity was also observed by looking at RIP1 phosphorylation itself. Indeed, we found that BV6 pre-treatment led to RIP1 mobility shifts following TNF stimulation (Figure 3e). $\lambda$-Phosphatase treatment of immunoprecipitated RIP1 confirmed that these modifications were due to phosphorylation (Figure 3f), and combined treatment with BV6 and $\mathrm{Nec}-1$ revealed that TNF-induced RIP1 phosphorylation in the presence of BV6 requires RIP1 kinase activity (Figure $3 \mathrm{~g}$ ). We conclude that CIAP1 depletion induces RIP1 kinase activity, which was shown to be required for TNF-induced necrosome formation. ${ }^{8,10}$

cIAP1 and TAK1 regulate the formation of the necrosome complex. Previous studies on tumor necrosis factor receptor 1 (TNFR1) signaling have shown that ClAPmediated Lys63-ubiquitination of RIP1 protects cells from apoptosis by inducing formation of a TAK1-containing 
a
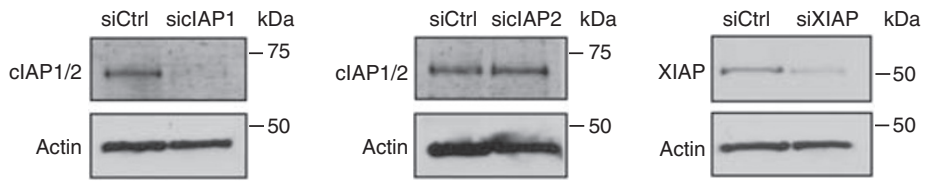

b

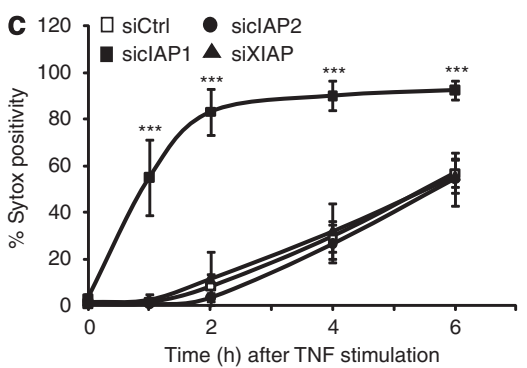

Figure 2 Loss of cIAP1 sensitizes L929 cells to TNF-induced necrosis. (a) Protein levels of cIAP1, clAP2, and XIAP were repressed by using RNAi. Knockdown efficiency was checked by western blot. Detection of clAP2 is below detection limit. (b) mRNA levels of clAP2 were tested using RT-PCR. cDNA from macrophages was included as a positive control. (c) L929 cells with repressed levels of cIAP1, cIAP2, or XIAP were stimulated with TNF (10000 IU/ml) for 2, 4, or $6 \mathrm{~h}$. Cell death (\% Sytox positivity) was analyzed by flow cytometry. Results are representative of at least three independent experiments. Error bars indicate standard deviation. ${ }^{* \star \star} P<0.001$ (Mann-Whitney U-test)

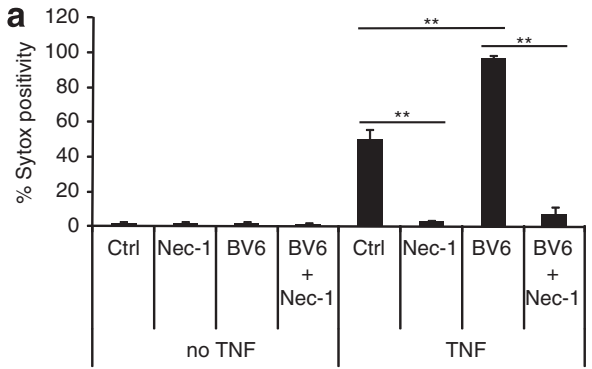

C
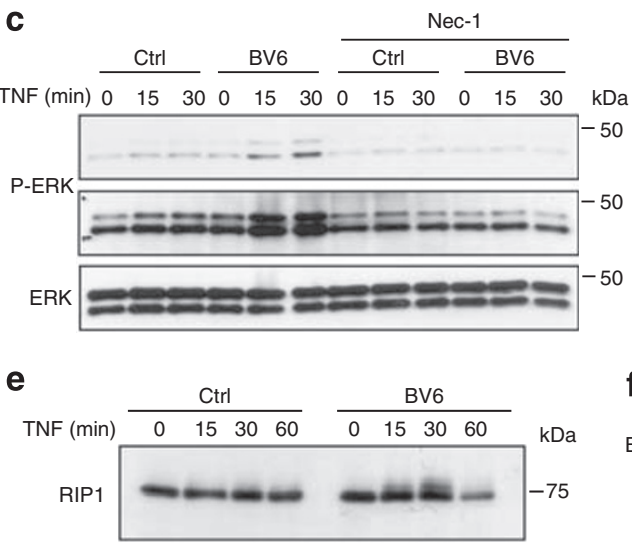

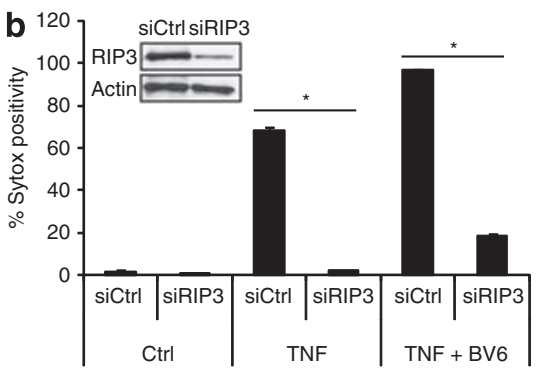

d
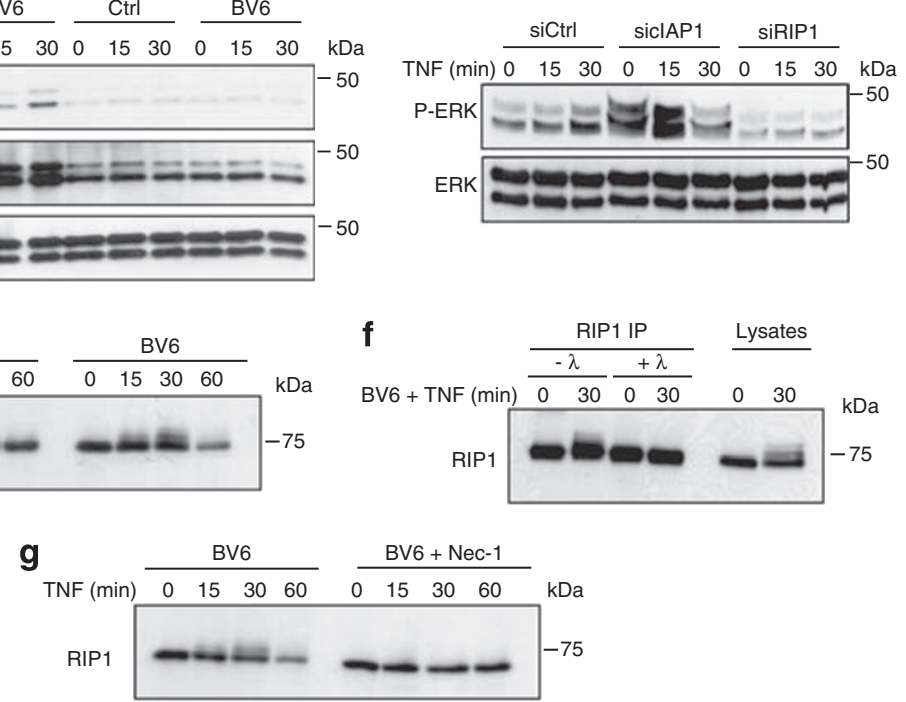

Figure 3 cIAP1 repression induces RIP1 kinase activity. (a) L929 cells were pre-treated with $10 \mu \mathrm{M} \mathrm{Nec-1}$ and $1 \mu \mathrm{M} \mathrm{BV} 6$ for $2 \mathrm{~h}$ and stimulated with TNF for $4 \mathrm{~h}$. (b) RIP3 levels in L929 were reduced by using RNAi. After $72 \mathrm{~h}$, cells were pre-treated with BV6 and stimulated with TNF for $6 \mathrm{~h}$. Results are representative of at least three independent experiments. Error bars indicate standard deviations. ${ }^{*} P<0.05$; ${ }^{*} P<0.01$ (Mann-Whitney U-test). (c) L929 cells were pre-treated with BV6 in the presence or absence of Nec-1. Next, cells were stimulated with TNF and lysed. ERK activation (phosphorylated ERK) was checked by western blot. (d) clAP1 and RIP1 levels were repressed using RNAi in L929 cells. Next, ERK phosphorylation was detected after TNF stimulation. Knockdown efficiency was checked by western blot. (e) L929 cells were pre-treated with BV6 and stimulated with TNF. (f) After TNF stimulation, RIP1 was immunoprecipitated and samples were treated with $\lambda$-phosphatase. (g) L929 cells were pre-treated with BV6 and Nec-1, followed by TNF stimulation. RIP1 protein levels were visualized using western blot 
pro-survival complex that prevents RIP1 from activating a caspase-8 death complex. ${ }^{16}$ Accordingly, CYLD, a Lys63-specific de-ubiquitinase, was shown to enhance TNF-induced apoptosis by stimulating formation of the RIP1-caspase-8 death complex. ${ }^{18}$ Recent studies have also shown that TNFR1-mediated necrosis requires the formation of a cytosolic necrosome complex containing RIP1, RIP3, and FADD. ${ }^{7,8,10}$ To investigate whether cIAP1 and TAK1 protect cells from TNFR1-induced necrotic death by preventing formation of this death complex, we first tested the effect of TAK1 repression in L929 cells. As shown in Figure 4a, TAK1 RNAi strongly sensitized cells to TNF-induced necrosis, and, importantly, this effect was abolished when RIP1 kinase activity was blocked by Nec-1. To test whether the protective effect of TAK1 requires its kinase activity, we pre-treated L929 cells with a derivative of the TAK1 kinase inhibitor 5Z-7-oxozeaenol (5Z-7). Like TAK1 depletion, use of $5 Z-7$ strongly sensitized L929 cells to TNF-induced necrosis, and this effect was also prevented by Nec-1 (Figure 4b). In parallel experiments, we found that lowering CYLD levels protected L929 cells against TNF-induced necrosis (Figure 4c). Next, we analyzed how depletion of ClAP1 with BV6 and inhibition of TAK1 kinase activity with 5Z-7 affect formation of the necrosome complex. As shown in Figure $4 d$ and $e$, both treatments led to increased recruitment of RIP1 to RIP3 and FADD following TNF stimulation. As previously reported by Cho et al., ${ }^{10}$ we found constitutive binding of RIP3 to FADD. Taken together, our data indicate that clAP1 and TAK1 protect cells from TNF-induced necrosis by preventing formation of the RIP1RIP3-FADD necrosome.

cIAP1 or TAK1 depletion augments RIP1/RIP3dependent ROS generation induced by TNF. RIP1/ RIP3-mediated cell death is thought to depend on the production of reactive oxygen species (ROS).$^{7,24,25}$ To determine whether sensitization of clAP1-depleted L929 cells to TNF-induced necrotic cell death involves generation of ROS or activation of an alternative death mechanism, we monitored ROS generation. As shown in Figure $5 a$ and $b$, TNF-induced RIP1/RIP3-dependent death was accompanied by modest intracellular ROS production, which was measured by incubating the cells with dihydrorhodamine 123 (DHR123). Interestingly, we found that BV6 treatment induced a rapid and massive production of ROS upon TNF stimulation; this ROS production was greatly repressed when cell death was inhibited by Nec-1 or by repressing RIP3 levels (Figure $5 a$ and $b$ ). Like BV6 treatment, lowering clAP1 or TAK1 levels by RNAi also considerably augmented ROS production in response to TNF, whereas repressing CYLD levels prevented ROS generation (Figure $5 c$ and $d$ ). As expected, XIAP knockdown had no effect on TNF-dependent ROS induction. To test whether the increased ROS generation observed in clAP1-depleted L929 cells was responsible for the sensitization to TNF-induced necrosis, we compared cell death in L929 cells treated with BV6 alone or in combination with the anti-oxidant butylated hydroxyanisole (BHA). BHA treatment greatly reduced BV6 sensitization to TNF-induced necrosis, which indicates that the peak of ROS production monitored in clAP1-depleted L929 cells was responsible for the increase in cell death (Figure 5e).

TNF-mediated ROS production can originate from the NADPH oxidase complex ${ }^{26}$ or at the mitochondria. ${ }^{5,25}$ As $\mathrm{BHA}$ is not only a broad ROS scavenger, but also a cytosolic phospholipase A2 inhibitor, ${ }^{27}$ we investigated the lethal contribution of each source by comparing the effects of their specific inhibition. A recent study reported that ROS generation requires recruitment of riboflavin kinase (RFK) and the NADPH oxidases Nox1 and Nox2 to TNFR1. ${ }^{26,28}$ We found that specific repression of components of the NADPH oxidase complex (RFK, Nox1, and p22phox) by RNAi did not affect death induced by TNF or TNF + BV6, but repression of NADH dehydrogenase (ubiquinone) 1 beta subcomplex 8 (NDUFB8) (subunit of mitochondrial complex I) strongly attenuated it (Figure $5 f$ and data not shown). Our results therefore indicate that, in the absence of clAP1, TNF-induced necrosis in L929 cells requires RIP1/3-mediated mitochondrial ROS production. We also found that absence of clAP1 greatly enhanced TNF-induced ROS production without inducing translocation of RIP1 or RIP3 to the mitochondria; this points to the involvement of other cytoplasmic intermediates (Supplementary Figure 3). Taken together, our results show that clAP1 and TAK1 protect L929 cells from TNF-induced necrosis by repressing RIP1-kinase-dependent induction of ROS generation and cell death.

\section{Discussion}

The identification of the RIP1 kinase inhibitor $\mathrm{Nec}-1$ has enabled researchers to reveal the involvement of necrotic cell death in an increasing number of pathological conditions. ${ }^{3,4}$ Indeed, RIP1 kinase activity is dispensable in most apoptotic conditions, but is crucial for the activation of a regulated form of necrosis recently named necroptosis. ${ }^{4,29}$ In the absence of an in vivo genetic model of kinase-inactive RIP1, the use of Nec-1 has emerged as the best tool for studying the function of RIP1 kinase. So far, necrotic cell death has been implicated in neuronal toxicity, ischemic brain injury, myocardial infarction, chemotherapy-induced cell death, and during viral infection. ${ }^{1,10}$ The finding that RIP1 is implicated in both apoptotic and necrotic pathways suggests that these cell death processes, which were initially defined as being mutually exclusive, might share similar regulatory mechanisms. ${ }^{18}$ IAP family members protect cells from apoptosis by inhibiting caspases and by regulating RIP1 ubiquitination status. $^{12,13,16,30,31}$ In addition, IAPs have been implicated in several RIP1-dependent apoptotic triggers (such as stimulation of TNFR1, Fas, or toll-like receptor 3 (TLR3)) ${ }^{14-16,18,32-34}$ that can also induce necrotic cell death under certain conditions. We found that the IAP antagonist BV6 greatly sensitized L929 cells to TNF-induced necrotic cell death, but not to necrosis induced by poly(I:C) + IFN $\beta$, anti-Fas + zVAD$\mathrm{fmk}$, or $\mathrm{H}_{2} \mathrm{O}_{2}$. We confirmed those results using an RNAi approach and identified clAP1 as a major regulator of TNFinduced necrosis. With the exception of $\mathrm{H}_{2} \mathrm{O}_{2}$, all the abovementioned necrotic triggers require intact RIP1 kinase activity (repressed by Nec-1). 5,9,20 Therefore, our results show the involvement of clAP1 only in a subset of RIP1-dependent necrotic pathways in L929 cells. 


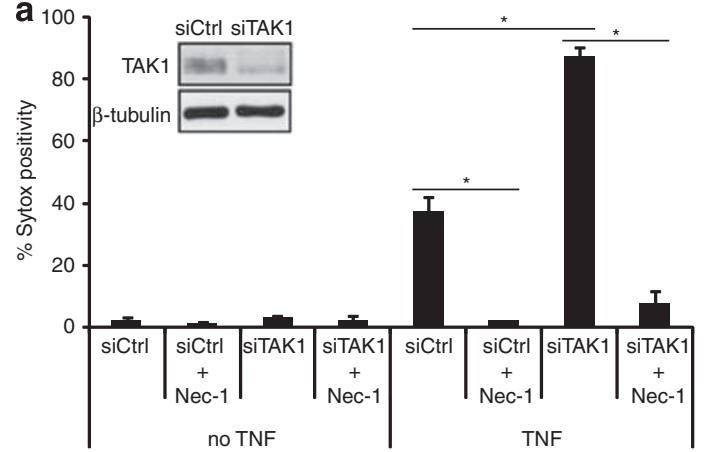

C

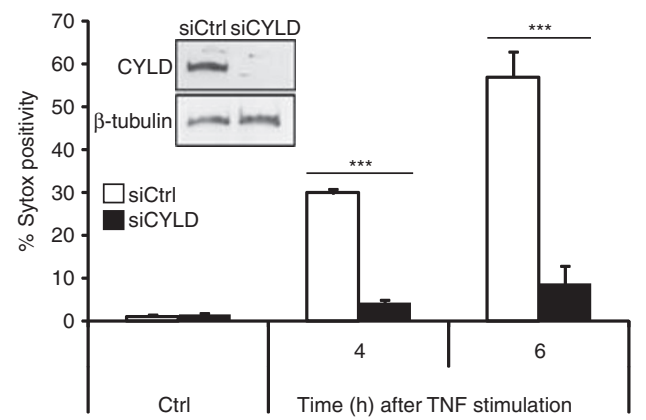

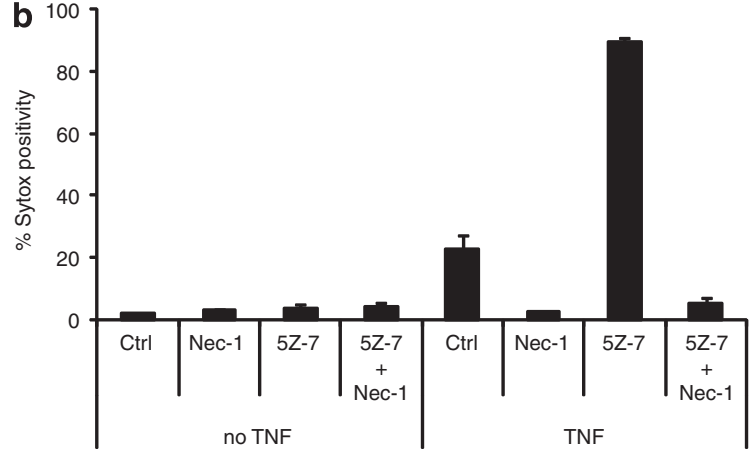

d
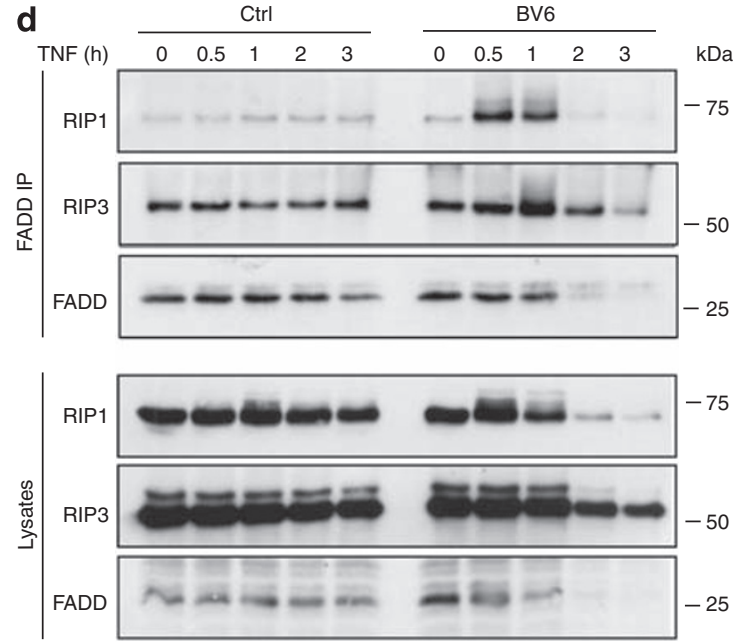

e

5Z-7
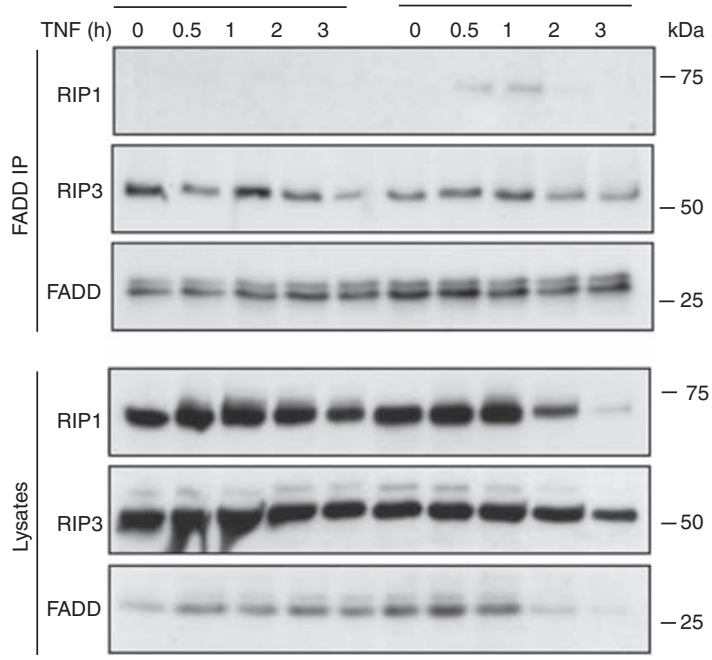

Figure 4 cIAP1 and TAK1 regulate the formation of the necrosome complex. (a) TAK1 levels in L929 were repressed by using RNAi. After $72 \mathrm{~h}$, cells were pre-treated with Nec-1 and triggered with TNF. (b) L929 cells were pre-treated with Nec-1 $(10 \mu \mathrm{M})$ and TAK1 kinase inhibitor $5 Z-7(1 \mu \mathrm{M})$ for $1 \mathrm{~h}$, and then stimulated with TNF $(10000 \mathrm{IU} / \mathrm{ml})$ for $2 \mathrm{~h}$. (c) CYLD levels were reduced in L929 cells as described above, and then stimulated with TNF for 4 or $6 \mathrm{~h}$. Cell death (\% Sytox positivity) was analyzed by flow cytometry. Knockdown efficiency was checked by western blot. Results are representative of at least three independent experiments. Error bars indicate standard deviations. ${ }^{\star} P<0.05 ;{ }^{* \star \star} P<0.001$ (Mann-Whitney U-test). (d and e) L929 cells were pre-treated with BV6 or 5Z-7, followed by TNF stimulation. FADD was immunoprecipitated and immunoblotted

Geserick et al. ${ }^{32}$ recently reported that loss of clAPs sensitizes a keratinocyte cell line to FasL-induced apoptosis. Intriguingly, in these cells, the presence of IAP antagonist allows moderate induction of necrosis in conditions where caspases are inhibited, which apparently does not occur in the absence of IAP antagonist. This observation indicates that the presence of clAPs negatively regulates FasL-induced necrosis. Similarly, the authors showed that mouse embryonic 


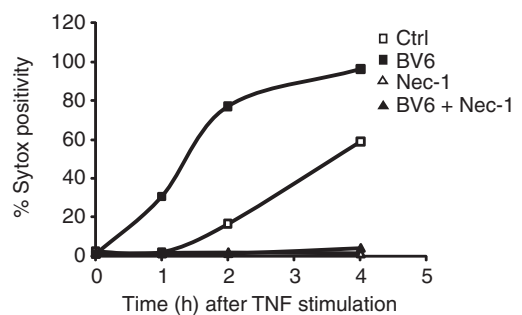

b

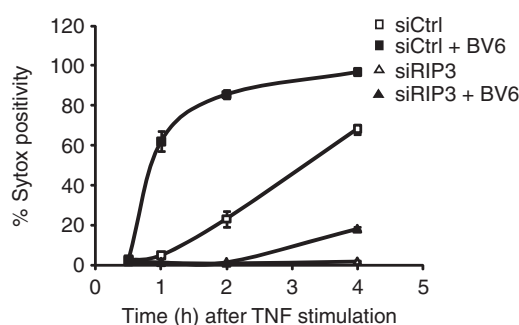

C

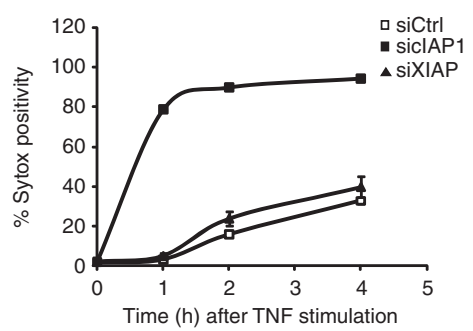

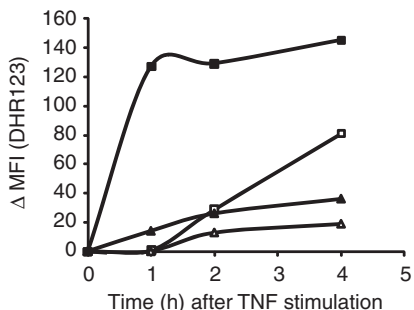
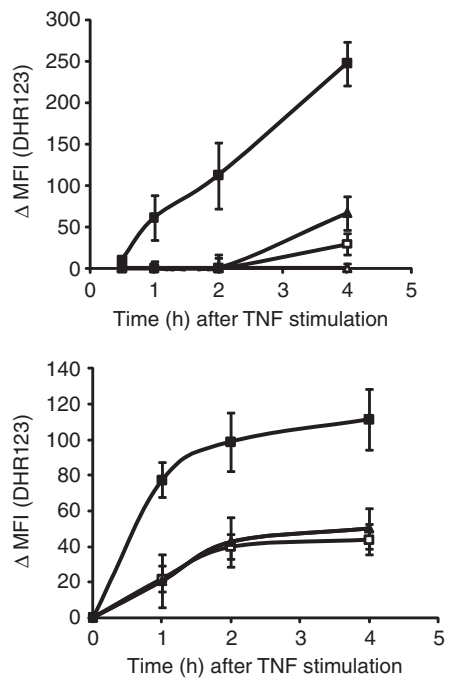
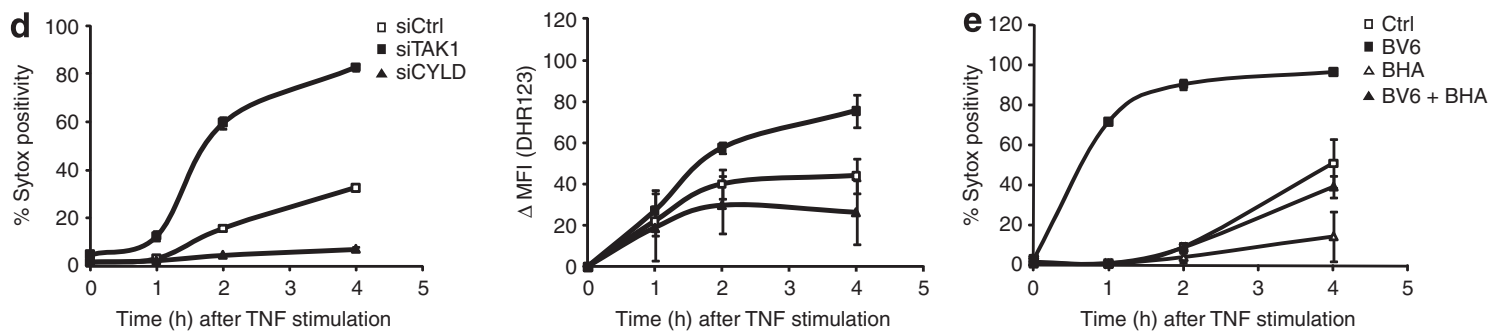

$\mathbf{f}$
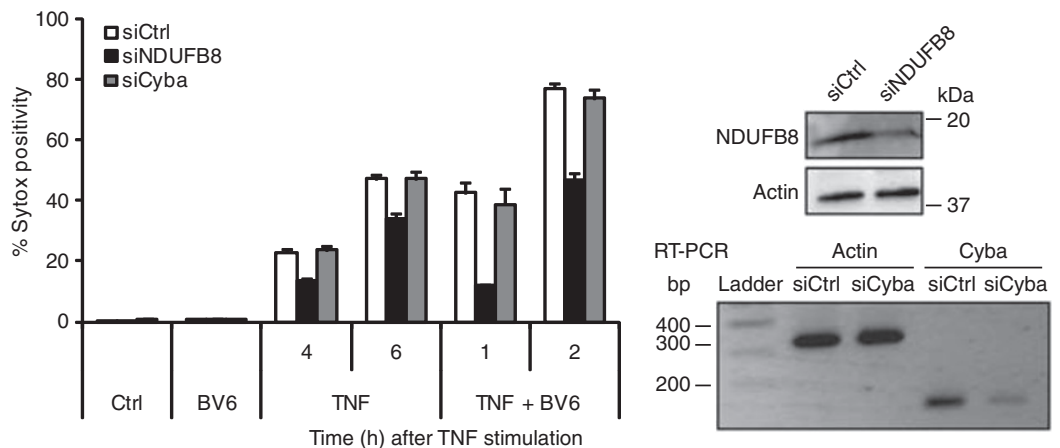

Figure 5 CIAP1 and TAK1 depletion augments RIP1/RIP3-dependent ROS generation induced by TNF. (a) L929 cells were pre-treated with BV6 and Nec-1, and then stimulated with TNF (10000 IU/ml). (b) RIP3 levels in L929 cells were reduced for $72 \mathrm{~h}$ using RNAi, and then treated with BV6 and TNF. Protein levels of (c) clAP1 or XIAP and (d) TAK1 or CYLD were reduced by using RNAi, followed by TNF stimulation. (e) L929 cells were pre-treated with BV6 and $100 \mu$ M BHA. (f) p22phox (encoded by the Cyba gene) and Ndufb8 levels were repressed using RNAi, followed by BV6 treatment and TNF stimulation. Knockdown efficiency was checked by western blot or RT-PCR. Cell death (\% Sytox positivity) and ROS generation ( $\triangle \mathrm{MFI}$ (DHR123)) were analyzed simultaneously by flow cytometry. Results are representative of at least two independent experiments. Error bars indicate standard deviations

fibroblasts depleted of IAPs are sensitized to a type of FasL-induced cell death that can only be blocked by combining Nec-1 with caspase inhibition, suggesting necrotic cell death. In our L929 cell system, triggering Fas induces apoptosis that is not sensitized by BV6 treatment (data not shown). Pure necrotic cell death is observed in L929 cells when 
Fas is triggered in the presence of zVAD-fmk, and again BV6 treatment had no impact on cell viability (Figure 1). This indicates no protective role of the clAPs in either Fasmediated apoptotic or necrotic cell death. The observed difference between our results and those of Geserick et al. might be explained by differential time kinetics of cell death induction (2-3h versus $24-48 \mathrm{~h}$, which leaves no room for sensitization in the case of L929 cells) or by the use of different triggers (FasL versus agonistic Fas receptor antibodies), different IAP antagonists (Compound A versus BV6), and different cell types. Moreover, MEFs are poorly sensitive to Fas-induced death; this is only revealed in sensitizing conditions, such as the addition of cycloheximide or IAP inhibitors, showing again a major difference from the L929 model system. Nevertheless, together with previous studies, our results suggest that ubiquitination of RIP1 prevents it from activating death pathways. The finding that clAPs act as E3 ubiquitin ligases for RIP1 downstream of TNFR1 explains why clAPsdepleted cells are greatly sensitized to TNF-induced death. The absence of BV6-induced sensitization when stimulating L929 cells with TLR3 or Fas agonists could indicate that other E3 ubiquitin ligases confer the ubiquitin-dependent protective effect on RIP1, potentially in a cell-type-specific manner. This hypothesis is consistent with the recent findings of Chang et al., ${ }^{35}$ who reported that pellino 1 acts as an E3 ubiquitin ligase for RIP1 in TLR3 signaling.

The fate of polyubiquitinated proteins is determined largely by the nature of the ubiquitin linkages. Generally, addition of Lys48-polyubiquitin chains allows recognition and degradation by the proteasome, whereas Lys63-ubiquitination has emerged as a key regulatory event that conveys signals from receptors to intracellular kinase cascades. cIAP-mediated Lys63-ubiquitination of RIP1 has been shown to protect cells from TNF-induced apoptosis by allowing the formation of a TAK1-containing pro-survival complex that prevents RIP1 from activating caspase-8-dependent death. ${ }^{16}$ Accordingly, the Lys63-specific de-ubiquitinase activity of CYLD was proposed to enhance TNF-induced apoptosis by reverting the effects of clAPs. ${ }^{18}$ Here, using Nec-1- and RIP3-specific RNAi, we positioned clAP1 and TAK1 upstream or at the level of RIP1/3, and found that repressing either protein greatly sensitized L929 cells to TNF-induced necrosis, whereas lowering CYLD levels had the opposite effect. Our attempts to detect TNF-induced RIP1 ubiquitination failed, most probably due to the low levels of ubiquitinated RIP1 in L929 cells. However, our results suggest that cIAP1 protects L929 cells from TNF-induced necrosis through Lys63-ubiquitination of RIP1. It is tempting to speculate that Lys63-ubiquitinated RIP1 allows the formation of a TAK1-containing pro-survival complex that represses RIP1 kinase activity. Consistent with this hypothesis, we found that RIP1 kinase-dependent ERK phosphorylation induced by TNF is greatly increased when clAP1 expression is repressed. In accordance with previous results, we also found that blocking ERK phosphorylation had no significant inhibitory effect on BV6-induced sensitization of TNF-induced necrosis. ${ }^{24}$ Interestingly, we found that pretreating L929 cells with BV6 led to TNF-induced RIP1 kinasedependent phosphorylation of RIP1 at a time that coincided with the recruitment of RIP1 to RIP3 and FADD. Further research is required to identify the BV6-induced phosphory- lation sites on RIP1, which might be implicated in the formation of the RIP1 and RIP3 complex and induction of necrotic cell death.

Several studies report that RIP1/RIP3-regulated necrosis is ROS mediated, and we confirmed strong correlations between cell death and RIP3- or RIP1-kinase-dependent ROS induction. For this reason, we investigated whether loss of clAP1 influences ROS production. Reducing clAP1 concentration did not affect basal levels of ROS. However, upon TNF stimulation, ROS generation increased considerably, and this effect was repressed by Nec-1 and by knocking down RIP3. Interestingly, lowering CYLD levels also inhibited ROS induction, suggesting that the RIP1 ubiquitination status determines its kinase-dependent capacity to induce ROS. Although controversial, the ability of RIP1 to induce NF- $\kappa$ B activation was shown to rely on its ubiquitination status, with Lys63-ubiquitin chains serving as a scaffold for the recruitment of TAK1 and for the activation of the IKK complex. ${ }^{36-38}$ We found that repressing TAK1 levels or inhibiting its kinase activity sensitized cells to TNF-induced necrosis and led to increased ROS generation. Therefore, clAP1 deficiency might increase TNF-induced ROS generation owing to the absence of NF- $\kappa \mathrm{B}$ activation. Indeed, clAPs are E3's for RIP1 ${ }^{16,39}$ and play a crucial role in TNF-induced $\mathrm{NF}-\kappa \mathrm{B}$ activation, which is known to upregulate the antioxidative capacity of the cell. ${ }^{40}$ However, our results negate this possibility. Indeed, we found that $\mathrm{Nec}-1$ prevents BV6induced TNF-dependent sensitization to death and reduces ROS generation without restoring NF- $\kappa \mathrm{B}$ capacities (data not shown). These data are consistent with previous studies reporting that NF- $\kappa \mathrm{B}$ does not protect against TNF-induced necrosis. ${ }^{41}$ Taken together, our results show that NF- $\kappa$ B inhibition is not sufficient to explain the massive TNF-dependent ROS production and suggest that cIAP1, TAK1 and CYLD play a direct role in regulating $R O S$ production by modulating RIP1 kinase activity through ubiquitination.

In conclusion, this work complements and extends data from a recent publication by $\mathrm{He}$ et al., who found increased TNF-dependent necrotic cell death in cells treated with a combination of IAP antagonist (smac mimetic) and zVAD-fmk. We show that necrotic and apoptotic cell death share some regulatory components and mechanisms. We also describe a new function for clAP1 and TAK1 in protecting cells from TNF-induced necrosis by regulating RIP1 kinasedependent generation of ROS.

\section{Materials and Methods}

Cell lines. L929sAhFas cells had been generated by expressing the human Fas gene in L929sA cells, a TNF-sensitive derivative of the murine fibrosarcoma cell line L929. ${ }^{2}$ These cells are referred to as L929 cells and were cultured in Dulbecco's modified Eagle's medium supplemented with $10 \%$ fetal calf serum, penicillin $(100 \mathrm{lU} / \mathrm{ml})$, streptomycin $(0.1 \mathrm{mg} / \mathrm{ml})$, and L-glutamine $(0.03 \%)$. Human Jurkat clones deficient in FADD were a gift from Dr. J Blenis and were cultured in RPMI 1640 medium supplemented with $10 \%$ fetal calf serum, $1 \mathrm{mM}$ L-glutamine, $25 \mathrm{mM}$ HEPES buffer, $50 \mathrm{U} / \mathrm{ml}$ penicillin, and $50 \mu \mathrm{g} / \mathrm{ml}$ streptomycin.

Antibodies, cytokines, and reagents. Recombinant human TNF, produced and purified to at least $99 \%$ homogeneity in our laboratory, has a specific biological activity of $3 \times 10^{7} \mathrm{IU} / \mathrm{mg}$ and is used for stimulation of L929 cells. FADD-1- Jurkat cells were stimulated with human TNF (Biochrom AG, Berlin, Germany). Anti-human Fas antibodies (clone 2R2; Cell Diagnostica, Munster, 
Germany) and poly(l:C) (synthetic dsRNA) (Amersham Pharmacia Biotech, Rainham, UK) were used at $125 \mathrm{ng} / \mathrm{ml}$ and $3.5 \mu \mathrm{g} / \mathrm{ml}$, respectively. Recombinant murine IFN $\beta$, produced in Escherichia coli and purified in our laboratory, was used at $1000 \mathrm{IU} / \mathrm{ml}$. The caspase peptide inhibitor, ZVAD-fmk (Bachem, Bubendorf, Switzerland), was used at $10 \mu \mathrm{M}$. BHA, U0126, 3-(4,5-dimethylthiazol-2-yl)-2, 5-diphenyltetrazolium bromide (MTT), and $\mathrm{H}_{2} \mathrm{O}_{2} 30 \%$ (all from Sigma Aldrich, St. Louis, MO, USA) were used at, respectively, $100 \mu \mathrm{M}, 20 \mu \mathrm{M}, 500 \mu \mathrm{g} / \mathrm{ml}$, and $2 \mathrm{mM}$. Nec-1 (Calbiochem, San Diego, CA, USA) was used at $10 \mu \mathrm{M}$. IAP antagonist BV6 was used at $1 \mu \mathrm{M}$ (Genentech Inc., South San Francisco, CA, USA). A derivative of 5Z-7, NP-009245, was used at $1 \mu \mathrm{M}$ (AnalytiCon Discovery GmbH, Potsdam, Germany). Sytox Red Dead Cell Stain, DHR123 (Molecular Probes-Invitrogen, Eugene, OR, USA), and propidium iodide (Sigma Aldrich, Steinheim, Germany) were used at, respectively, $5 \mathrm{nM}, 1 \mu \mathrm{M}$, and $3 \mu \mathrm{M}$. The following antibodies were used for L929 cells: antibody against CIAP1 and CIAP2 (RIAP1 antibody, ${ }^{22}$ a kind gift from Dr. RG Korneluk, University of Ottawa, Canada), anti-XIAP monoclonal antibody (MBL International, Woburn, MA, USA), anti- $\beta$ tubulin (HRP) (Abcam, Cambridge, UK), anti- $\beta$-actin (clone C4; MP Biomedicals Europe N.V., Illkirch, France), antibody against full-length and processed murine caspase-3 (rabbit polyclonal antibody made in-house), anti-NDUFB8 (MS105) (Bio-Connect B.V., TE Huissen, The Netherlands), anti-p44/42 MAPK (ERK1/2), anti-phospho-p44/42 MAPK (ERK1/2) (Thr202/Tyr204) (both Cell Signaling Technology, Beverly, MA, USA), anti-TAK1 (M-579), anti-CYLD (CYLD 1 (E-10)) (both from Santa Cruz Biotechnology, Santa Cruz, CA, USA), anti-RIP1 (610459; BD Biosciences, Franklin Lakes, NJ, USA), anti-RIP3 (Sigma Aldrich), and purified mouse anti-cytochrome $c$ (BD Pharmingen, San Diego, CA, USA). In FADD-1Jurkat cells, we used anti-cIAP1 and anti-cIAP2 (both Santa Cruz Biotechnology, anti-XIAP monoclonal antibody (BD Pharmingen), and anti- $\beta$-actin antibody (BD Biosciences).

Immunoprecipitation. L929 cells were lysed in NP-40 buffer ( $150 \mathrm{mM} \mathrm{NaCl,}$ $1 \% \mathrm{NP}-40,10 \%$ glycerol, $10 \mathrm{mM}$ Tris $(\mathrm{pH} 8)$ ) containing complete, EDTA-free protease inhibitor cocktail tablets (no. 11873580001) and phosphatase inhibitor cocktail tablets (PhosSTOP, no. 04906837001) (both from Roche Diagnostics Belgium N.V., Vilvoorde, Belgium). FADD was immunoprecipitated using the antiFADD (M-19, sc-6036) (Santa Cruz Biotechnology) and revealed using anti-FADD 12E7 (obtained from Dr. Strasser, WEHI, Melbourne, Australia).

Analysis of cell survival, cell death, and ROS production. Cell death and ROS production were analyzed by flow cytometry on a dual-laser (488 and $635 \mathrm{~nm}$ ) FACSCalibur with Cellquest software or a triple-laser $(405,488$, and $635 \mathrm{~nm}$ ) LSR-II with FACSDiva software (both from BD Biosciences). L929 cells were pretreated with IFN $\beta$ for $18 \mathrm{~h}, \mathrm{BV} 6$ for $2 \mathrm{~h}$, and zVAD-fmk, Nec-1, 5Z-7, BHA, or $\mathrm{U} 0126$ for $1 \mathrm{~h}$. TNF $(10000 \mathrm{IU} / \mathrm{ml}), \mathrm{H}_{2} \mathrm{O}_{2}(2 \mathrm{mM})$, poly(l:C) $(3.5 \mu \mathrm{g} / \mathrm{ml})$, or anti-Fas $(125 \mathrm{ng} / \mathrm{ml})$ was added for the indicated periods. Cell death or loss of plasma membrane integrity was determined by measuring Sytox Red or Pl-emitted fluorescence. Cellular ROS production was determined by measuring the conversion of DHR123 to R123. Only viable cells (Sytox negative) were gated for the analysis of ROS production. Values for ROS generation are expressed as $\triangle \mathrm{MFI}$ (DHR123) (median DHR123 fluorescence intensity value minus background value). All experiments were performed at least twice in triplicate. Cell survival was determined by MTT assays following standard protocol.

RNAi-mediated knockdown. L929 cells were transfected in six-well plates according to the manufacturer's protocol using $20 \mathrm{nM}$ siRNA targeting CIAP1, cIAP2, XIAP, RIP3, TAK1, CYLD, Cyba, or NDUFB8. As a negative control, we used siCONTROL non-targeting siRNA (ON-TARGETplus SMART pool siRNA; Dharmacon, Thermo Fisher Scientific, Waltham, MA, USA). INTERFERin (Polyplustransfection SA, Illkirch, France) was used as a transfection reagent. After $72 \mathrm{~h}$, L929 cells were stimulated with TNF and cell death was determined as described above. Knockdown efficiency was tested by western blot or RT-PCR.

RT-PCR. RNA was prepared from L929 cells using RNeasy Plus Mini Kit (Qiagen, Venlo, The Netherlands). Starting with $2 \mu \mathrm{g}$ RNA, cDNA was synthesized using SuperScript Reverse Transcriptase III kit (Invitrogen, Eugene, OR, USA). PCR was performed using GoTaq Green Mastermix (Promega, Fitchburg, WI, USA). PCR products were separated on $2 \%$ agarose gel and visualized by SYBR Safe DNA gel stain (Molecular Probes-Invitrogen).
Mitochondrial fractionation assay. Enrichment of cytosolic and mitochondrial fractions of L929 cells was performed according to the manufacturer's protocol (Mitochondrial/Cytosol Fractionation kit; BioVision, Mountain View, CA, USA). For each condition, $5 \times 10^{7}$ L929 cells were used.

\section{Conflict of Interest}

The authors declare no conflict of interest.

Acknowledgements. We thank Professor Wim Declercq and Dr. Saskia Lippens for critical feedback and discussion and Dr. A Bredan for editing. We are grateful to Dr. RG Korneluk for sending the RIAP1 antibody. TV and MB received a postdoctoral fellowship from the FWO, PB is paid by VIB, and NV obtained a predoctoral fellowship from the BOF, Ghent University. BL was a master student working in the labs of both Professor Simone Fulda and Professor Peter Vandenabeele. Research in the Vandenabeele group is supported by VIB, Ghent University, Research Foundation Flanders (FWO-Vlaanderen) (3G.0218.06 and G.0226.09), Federal Research Program IAP 6/18, European Research Program FP6 ApopTrain (MRTN-CT-035624) and FP7 Apo-Sys 200767, and the GROUP-ID consortium of the UGent MRP initiative. PV holds a Methusalem Grant (BOF09/ 01M00709) from the Flemish Government. Research in the Fulda group is supported by the Deutsche Forschungsgemeinschaft, Federal Research Program IAP 6/18, European Research Program FP6 ApopTrain (MRTN-CT-035624) and FP7 Apo-Sys 200767.

1. Vandenabeele P, Galluzzi L, Vanden Berghe T, Kroemer G. Molecular mechanisms of necroptosis: an ordered cellular explosion. Nat Rev Mol Cell Biol 2010; 11: 700-714.

2. Vercammen D, Beyaert R, Denecker G, Goossens V, Van Loo G, Declercq W et al. Inhibition of caspases increases the sensitivity of L929 cells to necrosis mediated by tumor necrosis factor. J Exp Med 1998; 187: 1477-1485.

3. Degterev A, Hitomi J, Germscheid M, Ch'en IL, Korkina O, Teng X et al. Identification of RIP1 kinase as a specific cellular target of necrostatins. Nat Chem Biol 2008; 4: 313-321.

4. Degterev A, Huang Z, Boyce M, Li Y, Jagtap P, Mizushima N et al. Chemical inhibitor of nonapoptotic cell death with therapeutic potential for ischemic brain injury. Nat Chem Biol 2005; 1: 112-119.

5. Vanden Berghe T, Vanlangenakker N, Parthoens E, Deckers W, Devos M, Festjens N et al. Necroptosis, necrosis and secondary necrosis converge on similar cellular disintegration features. Cell Death Differ 2010; 17: 922-930.

6. Vanlangenakker N, Berghe TV, Krysko DV, Festjens N, Vandenabeele P. Molecular mechanisms and pathophysiology of necrotic cell death. Curr Mol Med 2008; 8: 207-220.

7. Zhang DW, Shao J, Lin J, Zhang N, Lu BJ, Lin SC et al. RIP3, an energy metabolism regulator that switches TNF-induced cell death from apoptosis to necrosis. Science 2009; 325: 332-336.

8. He S, Wang L, Miao L, Wang T, Du F, Zhao L et al. Receptor interacting protein kinase-3 (RIP3) determines cellular necrotic response to TNFa. Cell 2009; 137: 1100-1111.

9. Holler N, Zaru R, Micheau O, Thome M, Attinger A, Valitutti S et al. Fas triggers an alternative, caspase-8-independent cell death pathway using the kinase RIP as effector molecule. Nat Immunol 2000; 1: 489-495.

10. Cho Y, Challa S, Moquin D, Genga R, Ray TD, Guildford M et al. Phosphorylation-driven assembly of RIP1-RIP3 complex regulates programmed necrosis and virus-induced inflammation. Cell 2009; 137: 1112-1123.

11. Festjens N, Vanden Berghe T, Cornelis S, Vandenabeele P. RIP1, a kinase on the crossroads of a cell's decision to live or die. Cell Death Differ 2007; 14: 400-410.

12. Eckelman BP, Salvesen GS. The human anti-apoptotic proteins cIAP1 and cIAP2 bind but do not inhibit caspases. J Biol Chem 2006; 281: 3254-3260.

13. Choi YE, Butterworth M, Malladi S, Duckett CS, Cohen GM, Bratton SB. The E3 ubiquitin ligase CIAP1 binds and ubiquitinates caspases- 3 and -7 via unique mechanisms at distinct steps in their processing. J Biol Chem 2009; 284: 12772-12782.

14. Vince JE, Wong WW, Khan N, Feltham R, Chau D, Ahmed AU et al. IAP antagonists target ClAP1 to induce TNFalpha-dependent apoptosis. Cell 2007; 131: 682-693.

15. Varfolomeev E, Blankenship JW, Wayson SM, Fedorova AV, Kayagaki N, Garg P et al. IAP antagonists induce autoubiquitination of c-IAPs, NF-kappaB activation, and TNFalphadependent apoptosis. Cell 2007; 131: 669-681.

16. Bertrand MJ, Milutinovic S, Dickson KM, Ho WC, Boudreault A, Durkin J et al. clAP1 and CIAP2 facilitate cancer cell survival by functioning as E3 ligases that promote RIP1 ubiquitination. Mol Cell 2008; 30: 689-700.

17. Komander D. The emerging complexity of protein ubiquitination. Biochem Soc Trans 2009; 37: 937-953.

18. Wang $L$, Du $F$, Wang $X$. TNF-alpha induces two distinct caspase-8 activation pathways. Cell 2008; 133: 693-703. 
19. Vercammen D, Brouckaert G, Denecker G, Van de Craen M, Declercq W, Fiers W et al. Dual signaling of the Fas receptor: initiation of both apoptotic and necrotic cell death pathways. J Exp Med 1998; 188: 919-930.

20. Kalai M, Van Loo G, Vanden Berghe T, Meeus A, Burm W, Saelens X et al. Tipping the balance between necrosis and apoptosis in human and murine cells treated with interferon and dsRNA. Cell Death Differ 2002; 9: 981-994.

21. Varfolomeev E, Alicke B, Elliott JM, Zobel K, West K, Wong H et al. XIAP regulates cell death induction by pro-apoptotic receptor agonists. J Biol Chem 2009; 284: 34553-34560.

22. Holcik M, Lefebvre CA, Hicks K, Korneluk RG. Cloning and characterization of the rat homologues of the inhibitor of apoptosis protein 1, 2, and 3 genes. BMC Genom 2002; $3: 5$.

23. Devin A, Lin Y, Liu ZG. The role of the death-domain kinase RIP in tumour-necrosis-factorinduced activation of mitogen-activated protein kinases. EMBO Rep 2003; 4: 623-627.

24. Lin Y, Choksi S, Shen HM, Yang QF, Hur GM, Kim YS et al. Tumor necrosis factor-induced nonapoptotic cell death requires receptor-interacting protein-mediated cellular reactive oxygen species accumulation. J Biol Chem 2004; 279: 10822-10828.

25. Schulze-Osthoff K, Bakker AC, Vanhaesebroeck B, Beyaert R, Jacob WA, Fiers W. Cytotoxic activity of tumor necrosis factor is mediated by early damage of mitochondria functions. Evidence for the involvement of mitochondrial radical generation. J Biol Chem 1992; 267: 5317-5323.

26. Kim YS, Morgan MJ, Choksi S, Liu ZG. TNF-induced activation of the Nox1 NADPH oxidase and its role in the induction of necrotic cell death. Mol Cell 2007; 26: 675-687.

27. Festjens N, Kalai M, Smet J, Meeus A, Van Coster R, Saelens X et al. Butylated hydroxyanisole is more than a reactive oxygen species scavenger. Cell Death Differ 2006; 13: 166-169.

28. Yazdanpanah B, Wiegmann K, Tchikov V, Krut O, Pongratz C, Schramm M et al. Riboflavin kinase couples TNF receptor 1 to NADPH oxidase. Nature 2009; 460: 1159-1163.

29. Kroemer G, Galluzzi L, Vandenabeele P, Abrams J, Alnemri ES, Baehrecke EH et al. Classification of cell death: recommendations of the Nomenclature Committee on Cell Death 2009. Cell Death Differ 2009; 16: 3-11.

30. Varfolomeev E, Goncharov T, Fedorova AV, Dynek JN, Zobel K, Deshayes K et al. c-IAP1 and C-IAP2 are critical mediators of tumor necrosis factor alpha (TNFalpha)-induced NF-kappaB activation. J Biol Chem 2008; 283: 24295-24299.
31. Mahoney DJ, Cheung HH, Mrad RL, Plenchette S, Simard C, Enwere E et al. Both cIAP1 and cIAP2 regulate TNFalpha-mediated NF-kappaB activation. Proc Natl Acad Sci USA 2008; 105: 11778-11783.

32. Geserick P, Hupe M, Moulin M, Wong WW, Feoktistova M, Kellert B et al. Cellular IAPs inhibit a cryptic CD95-induced cell death by limiting RIP1 kinase recruitment. J Cell Biol 2009; 187: 1037-1054.

33. Weber A, Kirejczyk Z, Besch R, Potthoff S, Leverkus M, Hacker G. Proapoptotic signalling through Toll-like receptor-3 involves TRIF-dependent activation of caspase-8 and is under the control of inhibitor of apoptosis proteins in melanoma cells. Cell Death Differ 2010; 17 942-951.

34. Friboulet L, Pioche-Durieu C, Rodriguez S, Valent A, Souquere S, Ripoche H et al. Recurrent overexpression of C-IAP2 in EBV-associated nasopharyngeal carcinomas: critical role in resistance to Toll-like receptor 3-mediated apoptosis. Neoplasia 2008; 10: 1183-1194.

35. Chang M, Jin W, Sun SC. Peli1 facilitates TRIF-dependent Toll-like receptor signaling and proinflammatory cytokine production. Nat Immunol 2009; 10: 1089-1095

36. Wong WW, Gentle IE, Nachbur U, Anderton H, Vaux DL, Silke J. RIPK1 is not essential for TNFR1-induced activation of NF-kappaB. Cell Death Differ 2010; 17: 482-487.

37. Kelliher MA, Grimm S, Ishida Y, Kuo F, Stanger BZ, Leder P. The death domain kinase RIP mediates the TNF-induced NF-kappaB signal. Immunity 1998; 8: 297-303.

38. Wang C, Deng L, Hong M, Akkaraju GR, Inoue J, Chen ZJ. TAK1 is a ubiquitin-dependent kinase of MKK and IKK. Nature 2001; 412: 346-351.

39. Park SM, Yoon JB, Lee TH. Receptor interacting protein is ubiquitinated by cellular inhibitor of apoptosis proteins (c-IAP1 and C-IAP2) in vitro. FEBS Lett 2004; 566: $151-156$

40. Kamata $\mathrm{H}$, Honda S, Maeda S, Chang L, Hirata H, Karin M. Reactive oxygen species promote TNFalpha-induced death and sustained JNK activation by inhibiting MAP kinase phosphatases. Cell 2005; 120: 649-661.

41. Vanden Berghe T, Kalai M, Denecker G, Meeus A, Saelens X, Vandenabeele P. Necrosis is associated with IL-6 production but apoptosis is not. Cell Signal 2006; 18. 328-335.

\section{Supplementary Information accompanies the paper on Cell Death and Differentiation website (http://www.nature.com/cdd)}

